\title{
Existence of Chaos for Nonlinear Schrödinger Equation Under Singular Perturbations
}

\author{
Y. Charles Li \\ Communicated by Charles Li, received January 25, 2004.
}

\begin{abstract}
The work $[\mathbf{1}]$ is generalized to the singularly perturbed nonlinear Schrödinger (NLS) equation of which the regularly perturbed NLS studied in $[\mathbf{1}]$ is a mollification. Specifically, the existence of Smale horseshoes and Bernoulli shift dynamics is established in a neighborhood of a symmetric pair of Silnikov homoclinic orbits under certain generic conditions, and the existence of the symmetric pair of Silnikov homoclinic orbits has been proved in [2]. The main difficulty in the current horseshoe construction is introduced by the singular perturbation $\epsilon \partial_{x}^{2}$ which turns the unperturbed reversible system into an irreversible system. It turns out that the equivariant smooth linearization can still be achieved, and the Conley-Moser conditions can still be realized.
\end{abstract}

\section{Contents}

1. Introduction 225

2. Equivariant Smooth Linearization 227

3. The Poincaré Map and Its Representation 228

4. The Fixed Points of the Poincaré Map $P$

5. Existence of Chaos 231

6. Numerical Evidence for the Generic Assumptions 236

References 237

\section{Introduction}

Consider the singularly perturbed nonlinear Schrödinger (NLS) equation,

$$
i q_{t}=q_{\zeta \zeta}+2\left[|q|^{2}-\omega^{2}\right] q+i \epsilon\left[q_{\zeta \zeta}-\alpha q+\beta\right],
$$

1991 Mathematics Subject Classification. Primary 35Q55, 35Q30; Secondary 37L10, 37L50.

Key words and phrases. Homoclinic orbits, chaos, Samle horseshoes, equivariant smooth linearization, Conley-Moser conditions.

This work is partially supported by a Guggenheim Fellowship. 


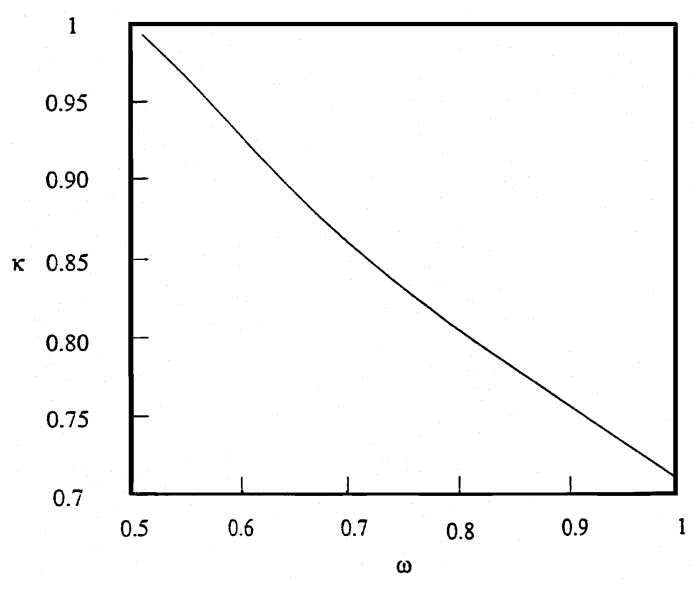

FiguRE 1. The graph of $\kappa(\omega)$.

where $q=q(t, \zeta)$ is a complex-valued function of the two real variables $t$ and $\zeta$, $t$ represents time, and $\zeta$ represents space. $q(t, \zeta)$ is subject to periodic boundary condition of period $2 \pi$, and even constraint, i.e.

$$
q(t, \zeta+2 \pi)=q(t, \zeta), \quad q(t,-\zeta)=q(t, \zeta) .
$$

$\alpha>0$ and $\beta>0$ are constants, and $\epsilon>0$ is the perturbation parameter. For simplicity of presentation, we restrict $\omega$ by $\omega \in(1 / 2,1)$. In [2], the following theorem on the existence of Silnikov homoclinic orbits was proved.

THEOREM 1.1. There exists a $\epsilon_{0}>0$, such that for any $\epsilon \in\left(0, \epsilon_{0}\right)$, there exists a codimension 1 surface in the external parameter space $(\alpha, \beta, \omega) \in \mathbb{R}^{+} \times \mathbb{R}^{+} \times$ $\mathbb{R}^{+}$where $\omega \in\left(\frac{1}{2}, 1\right) / S, S$ is a finite subset, and $\alpha \omega<\beta$. For any $(\alpha, \beta, \omega)$ on the codimension 1 surface, the singularly perturbed nonlinear Schrödinger equation (1.1) possesses a symmetric pair of Silnikov homoclinic orbits asymptotic to a saddle $Q_{\epsilon}$. The codimension 1 surface has the approximate representation given by $\alpha=$ $1 / \kappa(\omega)$, where $\kappa(\omega)$ is plotted in Figure 1.

Notice that if $q(t, \zeta)$ is a homoclinic orbit, then $q(t, \zeta+\pi)$ is another homoclinic orbit. Thus $q(t, \zeta)$ and $q(t, \zeta+\pi)$ form a symmetric pair of homoclinic orbits. Based upon the above theorem, we will construct Smale horseshoes in a neighborhood of the symmetric pair of homoclinic orbits. The construction is a generalization of that in $[\mathbf{1}]$ where the singular perturbation $\epsilon \partial_{x}^{2}$ is mollified into a bounded Fourier multiplier. The main difficulty in the current horseshoe construction is introduced by the singular perturbation $\epsilon \partial_{x}^{2}$ which turns the unperturbed reversible system into an irreversible system. Specifically, denote by $F_{\epsilon}^{t}$ the evolution operator of the singularly perturbed nonlinear Schrödinger equation (1.1). When $\epsilon=0, F_{0}^{t}$ is a group. When $\epsilon>0, F_{\epsilon}^{t}$ is only a semigroup. It turns out that the equivariant smooth linearization can still be achieved, and the Conley-Moser conditions can still be realized. Of course, one has to replace the inverse of the evolution operator $F_{\epsilon}^{t}$ by preimage. The article is organized as follows: In section 2, we present equivariant smooth linearization. In section 3 , we present the Poincaré map and its representation. In section 4 , the fixed points of the Poincaré map is studied. 
In section 5, we present the existence of chaos. Finally, in section 6, numerical evidence for the generic conditions is presented.

\section{Equivariant Smooth Linearization}

The symmetric pair of Silnikov homoclinic orbits is asymptotic to the saddle $Q_{\epsilon}=\sqrt{I} e^{i \theta}$, where

$$
I=\omega^{2}-\epsilon \frac{1}{2 \omega} \sqrt{\beta^{2}-\alpha^{2} \omega^{2}}+\cdots, \quad \cos \theta=\frac{\alpha \sqrt{I}}{\beta}, \quad \theta \in\left(0, \frac{\pi}{2}\right) .
$$

Its eigenvalues are

$$
\lambda_{n}^{ \pm}=-\epsilon\left[\alpha+n^{2}\right] \pm 2 \sqrt{\left(\frac{n^{2}}{2}+\omega^{2}-I\right)\left(3 I-\omega^{2}-\frac{n^{2}}{2}\right)},
$$

where $n=0,1,2, \cdots, \omega \in\left(\frac{1}{2}, 1\right)$, and $I$ is given in (2.1). The crucial points to notice are: (1). only $\lambda_{0}^{+}$and $\lambda_{1}^{+}$have positive real parts, $\operatorname{Re}\left\{\lambda_{0}^{+}\right\}<\operatorname{Re}\left\{\lambda_{1}^{+}\right\} ;(2)$. all the other eigenvalues have negative real parts among which the absolute value of $\operatorname{Re}\left\{\lambda_{2}^{+}\right\}=\operatorname{Re}\left\{\lambda_{2}^{-}\right\}$is the smallest; (3). $\left|\operatorname{Re}\left\{\lambda_{2}^{+}\right\}\right|<\operatorname{Re}\left\{\lambda_{0}^{+}\right\}$. Actually, items (2) and (3) are the main characteristics of Silnikov homoclinic orbits.

Lemma 2.1. For any fixed $\epsilon \in\left(0, \epsilon_{0}\right)$, let $E_{\epsilon}$ be the codimension 1 surface in the external parameter space, on which the symmetric pair of Silnikov homoclinic orbits are supported (cf: Theorem 1.1). For almost every $(\alpha, \beta, \omega) \in E_{\epsilon}$, the eigenvalues $\lambda_{n}^{ \pm}$(2.2) satisfy the nonresonance condition of Siegel type: There exists a natural number $s$ such that for any integer $n \geq 2$,

$$
\left|\Lambda_{n}-\sum_{j=1}^{r} \Lambda_{l_{j}}\right| \geq 1 / r^{s},
$$

for all $r=2,3, \cdots, n$ and all $l_{1}, l_{2}, \cdots, l_{r} \in \mathbb{Z}$, where $\Lambda_{n}=\lambda_{n}^{+}$for $n \geq 0$, and $\Lambda_{n}=\lambda_{-n-1}^{-}$for $n<0$.

Proof. The same proof as in [1] can be carried through here.

Thus, in a neighborhood of $Q_{\epsilon}$, the singularly perturbed NLS (1.1) is analytically equivalent to its linearization at $Q_{\epsilon}[\mathbf{3}]$. In terms of eigenvector basis, (1.1) can be rewritten as

$$
\begin{aligned}
\dot{x} & =-a x-b y+\mathcal{N}_{x}(\vec{Q}), \\
\dot{y} & =b x-a y+\mathcal{N}_{y}(\vec{Q}), \\
\dot{z}_{1} & =\gamma_{1} z_{1}+\mathcal{N}_{z_{1}}(\vec{Q}) \\
\dot{z}_{2} & =\gamma_{2} z_{2}+\mathcal{N}_{z_{2}}(\vec{Q}) \\
\dot{Q} & =L Q+\mathcal{N}_{Q}(\vec{Q})
\end{aligned}
$$

where $a=-\operatorname{Re}\left\{\lambda_{2}^{+}\right\}, b=\operatorname{Im}\left\{\lambda_{2}^{+}\right\}, \gamma_{1}=\lambda_{0}^{+}, \gamma_{2}=\lambda_{1}^{+} ; \mathcal{N}$ 's vanish identically in a neighborhood $\Omega$ of $\vec{Q}=0, \vec{Q}=\left(x, y, z_{1}, z_{2}, Q\right), Q$ is associated with the rest of eigenvalues, $L$ is given as

$$
L Q=-i Q_{\zeta \zeta}-2 i\left[\left(2\left|Q_{\epsilon}\right|^{2}-\omega^{2}\right) Q+Q_{\epsilon}^{2} \bar{Q}\right]+\epsilon\left[-\alpha Q+Q_{\zeta \zeta}\right],
$$

and $Q_{\epsilon}$ is given in (2.1). The following theorem on well-posedness is standard [4]. Let $F^{t}(0 \leq t<\infty)$ be the evolution operator of the singularly perturbed NLS (2.3), and $H^{s}$ be the Sobolev space. 
Theorem 2.2. For any $s \geq 1$, and any $\vec{Q}_{0} \in H^{s+2}, F^{t}\left(\vec{Q}_{0}\right) \in C^{0}\left([0, \infty) ; H^{s+2}\right) \cap$ $C^{1}\left([0, \infty) ; H^{s}\right)$. For any fixed $t \in[0, \infty), F^{t}$ is a $C^{2}$ map in $H^{s}$.

\section{The Poincaré Map and Its Representation}

Denote by $h_{k}(k=1,2)$ the symmetric pair of Silnikov homoclinic orbits. The symmetry $\sigma$ of half spatial period shifting has the new representation in terms of the new coordinates

$$
\sigma \circ\left(x, y, z_{1}, z_{2}, Q\right)=\left(x, y, z_{1},-z_{2}, \sigma \circ Q\right) .
$$

We have the following facts about the homoclinic orbits:

(1) The homoclinic orbits are classical solutions,

(2) As $t \rightarrow-\infty$, the homoclinic orbits are tangent to the positive $z_{1}$-axis at $\vec{Q}=0$.

The same proof as in $[\mathbf{1}]$ works here for item (2). Since $a$ is the smallest attracting rate, we assume that

- (A1). As $t \rightarrow+\infty$, the homoclinic orbits are tangent to the $(x, y)$-plane at $\vec{Q}=0$.

The Poincaré section is defined as in [1].

Definition 3.1. The Poincaré section $\Sigma_{0}$ is defined by the constraints:

$$
\begin{aligned}
& y=0, \eta \exp \{-2 \pi a / b\}<x<\eta ; \\
& 0<z_{1}<\eta,-\eta<z_{2}<\eta,\|Q\|<\eta ;
\end{aligned}
$$

where $\eta$ is a small parameter.

The horseshoes are going to be constructed on this Poincaré section. The auxiliary Poincaré section is defined differently from that in [1].

Definition 3.2. The Poincaré section $\Sigma_{1}$ is defined by the constraints:

$$
\begin{aligned}
& z_{1}=\eta, \quad-\eta<z_{2}<\eta, \\
& \sqrt{x^{2}+y^{2}}<\eta, \quad\|Q\|<\eta .
\end{aligned}
$$

The Poincaré map is defined as follows.

Definition 3.3. The Poincare map $P$ is defined as:

$$
P: U \subset \Sigma_{0} \mapsto \Sigma_{0}, \quad P=P_{1}^{0} \circ P_{0}^{1},
$$

where

$$
P_{0}^{1}: U_{0} \subset \Sigma_{0} \mapsto \Sigma_{1}, \quad \forall \vec{Q} \in U_{0}, \quad P_{0}^{1}(\vec{Q})=F^{t_{0}}(\vec{Q}) \in \Sigma_{1},
$$

and $t_{0}=t_{0}(\vec{Q})>0$ is the smallest time $t$ such that $F^{t}(\vec{Q}) \in \Sigma_{1}$, and

$$
P_{1}^{0}: U_{1} \subset \Sigma_{1} \mapsto \overline{\Sigma_{0}}\left(=\Sigma_{0} \cup \partial \Sigma_{0}\right), \quad \forall \vec{Q} \in U_{1}, \quad P_{1}^{0}(\vec{Q})=F^{t_{1}}(\vec{Q}) \in \overline{\Sigma_{0}},
$$

and $t_{1}=t_{1}(\vec{Q})>0$ is the smallest time $t$ such that $F^{t}(\vec{Q}) \in \overline{\Sigma_{0}}$. 
The map $P_{0}^{1}$ has the explicit representation: Let $\vec{Q}^{0}$ and $\vec{Q}^{1}$ be the coordinates on $\Sigma_{0}$ and $\Sigma_{1}$ respectively, $y^{0}=0$, and $z_{1}^{1}=\eta$, then $t_{0}=\frac{1}{\gamma_{1}} \ln \frac{\eta}{z_{1}^{0}}$, and

$$
\begin{aligned}
x^{1} & =\left(\frac{z_{1}^{0}}{\eta}\right)^{\frac{a}{\gamma_{1}}} x^{0} \cos \left[\frac{b}{\gamma_{1}} \ln \frac{\eta}{z_{1}^{0}}\right], \\
y^{1} & =\left(\frac{z_{1}^{0}}{\eta}\right)^{\frac{a}{\gamma_{1}}} x^{0} \sin \left[\frac{b}{\gamma_{1}} \ln \frac{\eta}{z_{1}^{0}}\right], \\
z_{2}^{1} & =\left(\frac{\eta}{z_{1}^{0}}\right)^{\frac{\gamma_{2}}{\gamma_{1}}} z_{2}^{0}, \\
Q^{1} & =e^{t_{0} L} Q^{0} .
\end{aligned}
$$

Let $\vec{Q}_{*}^{0}$ and $\vec{Q}_{*}^{1}$ be the intersection points of the homoclinic orbit $h_{1}$ with $\overline{\Sigma_{0}}$ and $\Sigma_{1}$ respectively. The discussion with respect to the other homoclinic orbit $h_{2}$ is the same. In a small neighborhood of $\vec{Q}_{*}^{1}$, the map $P_{1}^{0}$ has an approximate representation. By virtue of the fact that the homoclinic orbit $h_{1}$ is a classical solution, the Well-Posedness Theorem 2.2 implies that $F^{t}\left(\vec{Q}_{*}^{1}\right)$ is $C^{1}$ in $t$. Thus for $\vec{Q}^{1}$ in a small neighborhood of $\vec{Q}_{*}^{1}$,

$$
P_{1}^{0}\left(\vec{Q}^{1}\right)=P_{1}^{0}\left(\vec{Q}_{*}^{1}\right)+\mathcal{L}\left(\vec{Q}^{1}-\vec{Q}_{*}^{1}\right)+\mathcal{O}\left(\left\|\vec{Q}^{1}-\vec{Q}_{*}^{1}\right\|^{2}\right),
$$

where

$$
\mathcal{L}\left(\vec{Q}^{1}-\vec{Q}_{*}^{1}\right)=\partial_{\vec{Q}} F^{t_{1}}\left(\vec{Q}_{*}^{1}\right) \circ\left(\vec{Q}^{1}-\vec{Q}_{*}^{1}\right)+\partial_{t} F^{t_{1}}\left(\vec{Q}_{*}^{1}\right) \circ \frac{\partial t_{1}}{\partial \vec{Q}^{1}}\left(\vec{Q}_{*}^{1}\right) \circ\left(\vec{Q}^{1}-\vec{Q}_{*}^{1}\right),
$$

and $t_{1}=t_{1}\left(\vec{Q}^{1}\right)$ is defined by the constraint that the $y$-coordinate of $F^{t_{1}}\left(\vec{Q}^{1}\right)$ vanishes,

$$
F_{y}^{t_{1}}\left(\vec{Q}^{1}\right)=0
$$

Thus

$$
\partial_{\vec{Q}} F_{y}^{t_{1}}\left(\vec{Q}_{*}^{1}\right)+\partial_{t} F_{y}^{t_{1}}\left(\vec{Q}_{*}^{1}\right) \frac{\partial t_{1}}{\partial \vec{Q}^{1}}\left(\vec{Q}_{*}^{1}\right)=0
$$

i.e.

$$
\frac{\partial t_{1}}{\partial \vec{Q}^{1}}\left(\vec{Q}_{*}^{1}\right)=-\frac{1}{\partial_{t} F_{y}^{t_{1}}\left(\vec{Q}_{*}^{1}\right)} \partial_{\vec{Q}} F_{y}^{t_{1}}\left(\vec{Q}_{*}^{1}\right)
$$

Let $\overrightarrow{\tilde{Q}}^{0}=\vec{Q}^{0}-\vec{Q}_{*}^{0}$, and $\overrightarrow{\tilde{Q}}^{1}=\vec{Q}^{1}-\vec{Q}_{*}^{1}$, then $P_{1}^{0}$ has the approximate representation

$$
\left(\begin{array}{c}
\tilde{x}^{0} \\
\tilde{z}_{1}^{0} \\
\tilde{z}_{2}^{0} \\
\tilde{Q}^{0}
\end{array}\right)=\mathcal{C}\left(\begin{array}{c}
\tilde{x}^{1} \\
\tilde{y}^{1} \\
\tilde{z}_{2}^{1} \\
\tilde{Q}^{1}
\end{array}\right)+\Xi,
$$

where

$$
\Xi \sim \mathcal{O}\left(\left(\tilde{x}^{1}\right)^{2}+\left(\tilde{y}^{1}\right)^{2}+\left(\tilde{z}_{2}^{1}\right)^{2}+\left\|\tilde{Q}^{1}\right\|^{2}\right)
$$




$$
\mathcal{C}=\left(\begin{array}{cccc}
c_{11} & c_{12} & c_{13} & C_{14} \\
c_{21} & c_{22} & c_{23} & C_{24} \\
c_{31} & c_{32} & c_{33} & C_{34} \\
C_{41} & C_{42} & C_{43} & C_{44}
\end{array}\right),
$$

in which $c_{j l}(j, l=1,2,3)$ are real constants, $C_{j 4}(j=1,2,3,4)$ and $C_{4 l}(l=1,2,3)$ are linear operators.

\section{The Fixed Points of the Poincaré Map $P$}

As $t_{0} \rightarrow+\infty$, to the leading order, the fixed points of $P$ satisfy

$$
\left(\begin{array}{c}
\hat{x}^{0} \\
0 \\
0 \\
\hat{Q}^{0}
\end{array}\right)=\mathcal{C}\left(\begin{array}{c}
x_{*}^{0} \cos b t_{0} \\
x_{*}^{0} \sin b t_{0} \\
\hat{z}_{2}^{1} \\
0
\end{array}\right),
$$

where

$$
\hat{z}_{2}^{1}=e^{a t_{0}} \tilde{z}_{2}^{1}, \quad \hat{x}^{0}=e^{a t_{0}} \tilde{x}^{0}, \quad \hat{Q}^{0}=e^{a t_{0}} \tilde{Q}^{0} .
$$

Explicitly, the second and the third equations in (4.1) are:

$$
\begin{aligned}
& x_{*}^{0}\left[c_{21} \cos b t_{0}+c_{22} \sin b t_{0}\right]+c_{23} \hat{z}_{2}^{1}=0, \\
& x_{*}^{0}\left[c_{31} \cos b t_{0}+c_{32} \sin b t_{0}\right]+c_{33} \hat{z}_{2}^{1}=0 .
\end{aligned}
$$

LEMMA 4.1. $c_{23}$ and $c_{33}$ do not vanish simultaneously.

Proof. Notice that $W^{u}\left(Q_{\epsilon}\right)$ is two-dimensional, and intersects $\Sigma_{0}$ (or its extension to $\left.-\eta<z_{1}<\eta\right)$ into a one-dimensional curve with tangent vector

$$
v=\mathcal{C}\left(\begin{array}{l}
0 \\
0 \\
1 \\
0
\end{array}\right) \text {. }
$$

Notice also that for any $\vec{Q} \in h_{1}$,

$$
\operatorname{dim}\left\{\mathcal{T}_{\vec{Q}} W^{u}\left(Q_{\epsilon}\right) \cap \mathcal{T}_{\vec{Q}} W^{s}\left(Q_{\epsilon}\right)\right\}=1,
$$

where $\mathcal{T}_{\vec{Q}}$ denotes the tangent space at $\vec{Q}$. If $c_{23}$ and $c_{33}$ vanish simultaneously, then $v \in \mathcal{T}_{\vec{Q}_{*}^{0}} W^{s}\left(Q_{\epsilon}\right)$ which implies that

$$
\operatorname{dim}\left\{\mathcal{T}_{\vec{Q}_{*}^{0}} W^{u}\left(Q_{\epsilon}\right) \cap \mathcal{T}_{\vec{Q}_{*}^{0}} W^{s}\left(Q_{\epsilon}\right)\right\}=2
$$

which contradicts (4.3). The lemma is proved.

Let

$$
\Delta_{1}=c_{21} c_{33}-c_{31} c_{23}, \quad \Delta_{2}=c_{22} c_{33}-c_{32} c_{23} .
$$

We assume that

- (A2). $\Delta_{1}$ and $\Delta_{2}$ do not vanish simultaneously. 
Then (4.2) has infinitely many solutions:

$$
t_{0}^{(l)}=\frac{1}{b}\left[l \pi-\varphi_{1}\right], \quad l \in \mathbb{Z}
$$

where

$$
\varphi_{1}=\arctan \left\{\Delta_{1} / \Delta_{2}\right\}
$$

Without loss of generality, we assume $c_{23} \neq 0$. Then, solving Eqs.(4.2), we have

$$
\hat{z}_{2}^{(1, l)}=-x_{*}^{0}\left[c_{23}\right]^{-1}\left\{c_{21} \cos b t_{0}^{(l)}+c_{22} \sin b t_{0}^{(l)}\right\} .
$$

Solving (4.1), we have

$$
\begin{aligned}
& \hat{x}^{(0, l)}=x_{*}^{0}\left[c_{11} \cos b t_{0}^{(l)}+c_{12} \sin b t_{0}^{(l)}\right]+c_{13} \hat{z}_{2}^{(1, l)}, \\
& \hat{Q}^{(0, l)}=x_{*}^{0}\left[C_{41} \cos b t_{0}^{(l)}+C_{42} \sin b t_{0}^{(l)}\right]+C_{43} \hat{z}_{2}^{(1, l)} .
\end{aligned}
$$

Finally, by the implicit function theorem, there exist infinitely many fixed points of $P$, which have the approximate expressions given above $[\mathbf{1}]$. Specifically, we have

Theorem 4.2. The Poincaré map $P$ has infinitely many fixed points labeled by $l\left(l \geq l_{0}\right)$ :

where as $l \rightarrow+\infty$,

$$
t_{0}=t_{0, l}, \hat{x}^{0}=\hat{x}_{l}^{0}, \hat{Q}^{0}=\hat{Q}_{l}^{0}, \hat{z}_{2}^{1}=\hat{z}_{2, l}^{1}
$$

$$
\begin{aligned}
t_{0, l} & =\frac{1}{b}\left[l \pi-\varphi_{1}\right]+o(1), \\
\hat{x}_{l}^{0} & =\hat{x}^{(0, l)}+o(1), \\
\hat{Q}_{l}^{0} & =\hat{Q}^{(0, l)}+o(1), \\
\hat{z}_{2, l}^{1} & =\hat{z}_{2}^{(1, l)}+o(1),
\end{aligned}
$$

in which $\hat{x}^{(0, l)}, \hat{Q}^{(0, l)}$ and $\hat{z}_{2}^{(1, l)}$ are given in (4.6),(4.7),(4.5).

\section{Existence of Chaos}

One can construct Smale horseshoes in the neighborhoods of the fixed points of $P$.

Definition 5.1. For sufficiently large natural number $l$, we define slab $S_{l}$ in $\Sigma_{0}$ as follows:

$$
\begin{aligned}
S_{l} \equiv & \left\{\vec{Q} \in \Sigma_{0} \mid \eta \exp \left\{-\gamma_{1}\left(t_{0,2(l+1)}-\frac{\pi}{2 b}\right)\right\} \leq\right. \\
& \tilde{z}_{1}^{0}(\vec{Q}) \leq \eta \exp \left\{-\gamma_{1}\left(t_{0,2 l}-\frac{\pi}{2 b}\right)\right\}, \\
& \left|\tilde{x}^{0}(\vec{Q})\right| \leq \eta \exp \left\{-\frac{1}{2} a t_{0,2 l}\right\}, \\
& \left|\tilde{z}_{2}^{1}\left(P_{0}^{1}(\vec{Q})\right)\right| \leq \eta \exp \left\{-\frac{1}{2} a t_{0,2 l}\right\}, \\
& \left.\left\|\tilde{Q}^{1}\left(P_{0}^{1}(\vec{Q})\right)\right\| \leq \eta \exp \left\{-\frac{1}{2} a t_{0,2 l}\right\}\right\},
\end{aligned}
$$

where the notations $\tilde{x}^{0}(\vec{Q}), \tilde{z}_{2}^{1}\left(P_{0}^{1}(\vec{Q})\right)$, etc. denote the $\tilde{x}^{0}$ coordinate of the point $\vec{Q}$, the $\tilde{z}_{2}^{1}$ coordinate of the point $P_{0}^{1}(\vec{Q})$, etc.. 

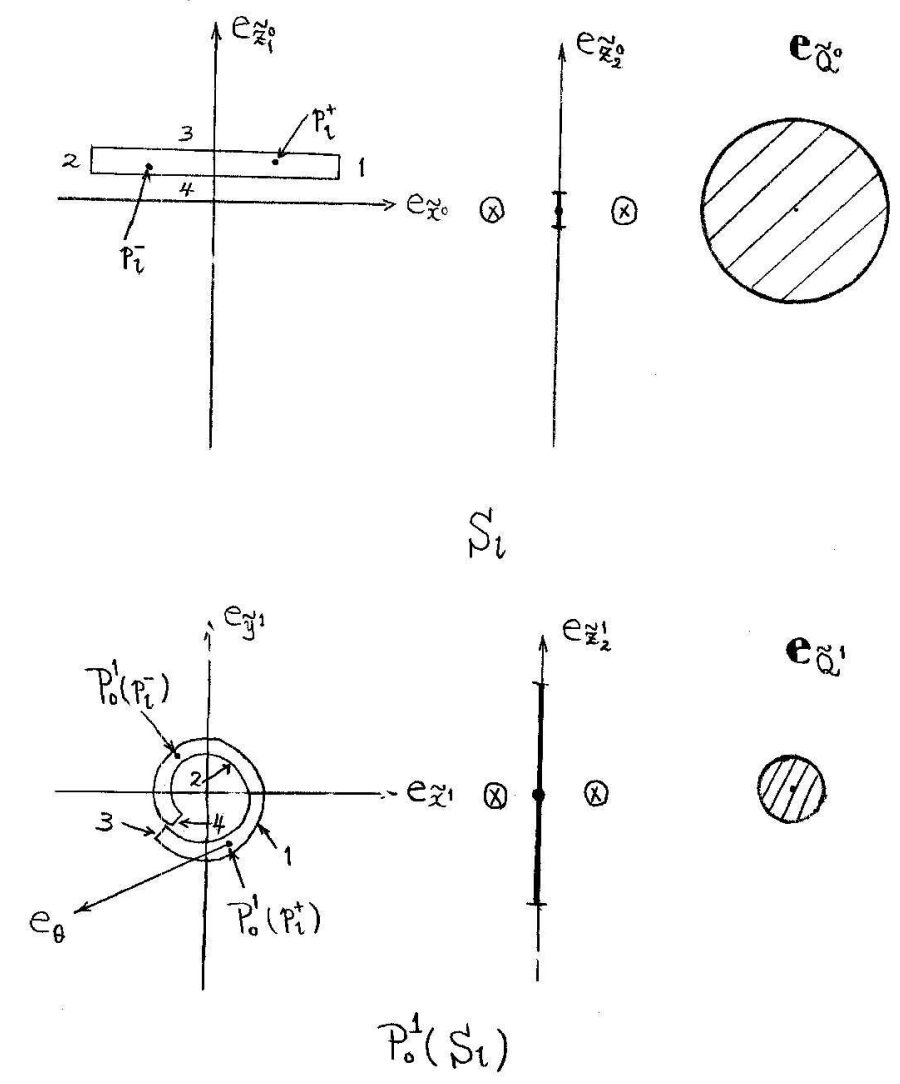

$\mathbf{e}_{\widetilde{Q}^{\prime}}$

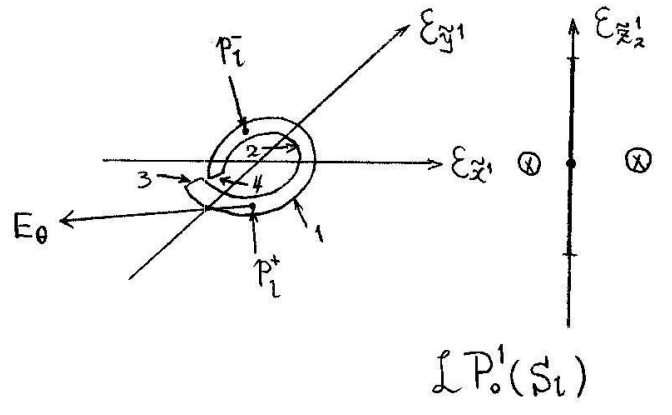

$\mathbf{E}_{\widetilde{Q}^{1}}$

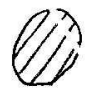

Figure 2. An illustration of $S_{l}, P_{0}^{1}\left(S_{l}\right)$, and $\mathcal{L} P_{0}^{1}\left(S_{l}\right)$.

$S_{l}$ is defined so that it includes two fixed points $p_{l}^{+}$and $p_{l}^{-}$of $P$ (Theorem 4.2). $S_{l}, P_{0}^{1}\left(S_{l}\right)$, and $\mathcal{L} P_{0}^{1}\left(S_{l}\right)$ are illustrated in Figure 2, where $\mathcal{L}$ is defined in (3.2). $\left\{e_{\tilde{x}^{0}}, e_{\tilde{z}_{1}^{0}}, e_{\tilde{z}_{2}^{0}}, \mathbf{e}_{\tilde{Q}^{0}}\right\}$ denotes the unit vectors along $\left(\tilde{x}^{0}, \tilde{z}_{1}^{0}, \tilde{z}_{2}^{0}, \tilde{Q}^{0}\right)$-directions in $\Sigma_{0}$, $\left\{e_{\tilde{x}^{1}}, e_{\tilde{y}^{1}}, e_{\tilde{z}_{2}^{1}}, \mathbf{e}_{\tilde{Q}^{1}}\right\}$ denotes the unit vectors along $\left(\tilde{x}^{1}, \tilde{y}^{1}, \tilde{z}_{2}^{1}, \tilde{Q}^{1}\right)$-directions in $\Sigma_{1}$, and under the linear map $\mathcal{L},\left\{e_{\tilde{x}^{1}}, e_{\tilde{y}^{1}}, e_{\tilde{z}_{2}^{1}}, \mathbf{e}_{\tilde{Q}^{1}}\right\}$ are mapped into $\left\{\mathcal{E}_{\tilde{x}^{1}}, \mathcal{E}_{\tilde{y}^{1}}, \mathcal{E}_{\tilde{z}_{2}^{1}}, \mathcal{E}_{\tilde{Q}^{1}}\right\}$. 

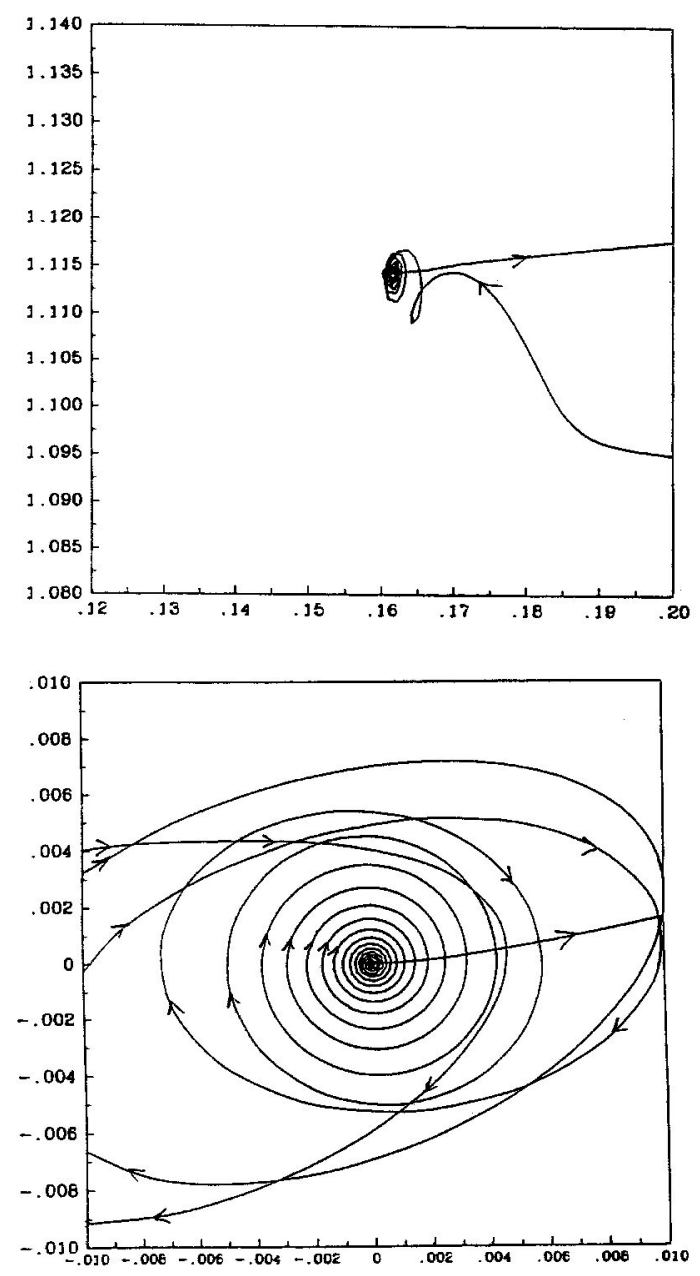

Figure 3. (a) shows one of the homoclinic orbits, and (b) shows the blow-up of the neighborhood of the saddle $Q_{\epsilon}$.

Let $e_{\theta}$ be the unit angular vector at $P_{0}^{1}\left(p_{l}^{+}\right)$of the polar coordinate frame on the $\left(\tilde{x}^{1}, \tilde{y}^{1}\right)$-plane. Let $E_{\theta}=\mathcal{L} e_{\theta}$, and we assume that

- (A3). $\operatorname{Span}\left\{e_{\tilde{x}^{0}}, \mathbf{e}_{\tilde{Q}^{0}}, E_{\theta}, \mathcal{E}_{\tilde{z}_{2}^{1}}\right\}=\Sigma_{0}$.

Let $S_{l, \sigma}=\sigma \circ S_{l}$ where the symmetry $\sigma$ is defined in (3.1). We need to define a larger slab $\hat{S}_{l}$ such that $S_{l} \cup S_{l, \sigma} \subset \hat{S}_{l}$. 
Definition 5.2. The larger slab $\hat{S}_{l}$ is defined as

$$
\begin{aligned}
\hat{S}_{l}= & \left\{\vec{Q} \in \Sigma_{0} \mid \eta \exp \left\{-\gamma_{1}\left(t_{0,2(l+1)}-\frac{\pi}{2 b}\right)\right\} \leq\right. \\
& z_{1}^{0}(\vec{Q}) \leq \eta \exp \left\{-\gamma_{1}\left(t_{0,2 l}-\frac{\pi}{2 b}\right)\right\}, \\
& \left|x^{0}(\vec{Q})-x_{*}^{0}\right| \leq \eta \exp \left\{-\frac{1}{2} a t_{0,2 l}\right\}, \\
& \left|z_{2}^{1}\left(P_{0}^{1}(\vec{Q})\right)\right| \leq\left|z_{2, *}^{1}\right|+\eta \exp \left\{-\frac{1}{2} a t_{0,2 l}\right\}, \\
& \left.\left\|Q^{1}\left(P_{0}^{1}(\vec{Q})\right)\right\| \leq \eta \exp \left\{-\frac{1}{2} a t_{0,2 l}\right\}\right\},
\end{aligned}
$$

where $z_{2, *}^{1}$ is the $z_{2}^{1}$-coordinate of $\vec{Q}_{*}^{1}$.

Definition 5.3. In the coordinate system $\left\{\tilde{x}^{0}, \tilde{z}_{1}^{0}, \tilde{z}_{2}^{0}, \tilde{Q}^{0}\right\}$, the stable boundary of $\hat{S}_{l}$, denoted by $\partial_{s} \hat{S}_{l}$, is defined to be the boundary of $\hat{S}_{l}$ along $\left(\tilde{x}^{0}, \tilde{Q}^{0}\right)$-directions, and the unstable boundary of $\hat{S}_{l}$, denoted by $\partial_{u} \hat{S}_{l}$, is defined to be the boundary of $\hat{S}_{l}$ along $\left(\tilde{z}_{1}^{0}, \tilde{z}_{2}^{0}\right)$-directions. A stable slice $V$ in $\hat{S}_{l}$ is a subset of $\hat{S}_{l}$, defined as the region swept out through homeomorphically moving and deforming $\partial_{s} \hat{S}_{l}$ in such a way that the part

$$
\partial_{s} \hat{S}_{l} \cap \partial_{u} \hat{S}_{l}
$$

of $\partial_{s} \hat{S}_{l}$ only moves and deforms inside $\partial_{u} \hat{S}_{l}$. The new boundary obtained through such moving and deforming of $\partial_{s} \hat{S}_{l}$ is called the stable boundary of $V$, which is denoted by $\partial_{s} V$. The rest of the boundary of $V$ is called its unstable boundary, which is denoted by $\partial_{u} V$. An unstable slice of $\hat{S}_{l}$, denoted by $H$, is defined similarly.

As shown in [1], under the assumption (A3), when $l$ is sufficiently large, $P\left(S_{l}\right)$ and $P\left(S_{l, \sigma}\right)$ intersect $\hat{S}_{l}$ into four disjoint stable slices $\left\{V_{1}, V_{2}\right\}$ and $\left\{V_{-1}, V_{-2}\right\}$ in $\hat{S}_{l} . V_{j}$ 's $(j=1,2,-1,-2)$ do not intersect $\partial_{s} \hat{S}_{l}$; moreover,

$$
\partial_{s} V_{i} \subset P\left(\partial_{s} S_{l}\right),(i=1,2) ; \partial_{s} V_{i} \subset P\left(\partial_{s} S_{l, \sigma}\right),(i=-1,-2) .
$$

Let

$$
H_{j}=P^{-1}\left(V_{j}\right), \quad(j=1,2,-1,-2),
$$

where and for the rest of this article, $P^{-1}$ denotes preimage of $P$. Then $H_{j}$ $(j=1,2,-1,-2)$ are unstable slices. More importantly, the Conley-Moser conditions are satisfied as shown in [1]. Specifically, Conley-Moser conditions are: Conley-Moser condition (i):

$$
\left\{\begin{array}{l}
V_{j}=P\left(H_{j}\right), \\
\partial_{s} V_{j}=P\left(\partial_{s} H_{j}\right), \quad(j=1,2,-1,-2) \\
\partial_{u} V_{j}=P\left(\partial_{u} H_{j}\right) .
\end{array}\right.
$$

Conley-Moser condition (ii): There exists a constant $0<\nu<1$, such that for any stable slice $V \subset V_{j}(j=1,2,-1,-2)$, the diameter decay relation

$$
d(\tilde{V}) \leq \nu d(V)
$$


holds, where $d(\cdot)$ denotes the diameter $[\mathbf{1}]$, and $\tilde{V}=P\left(V \cap H_{k}\right), \quad(k=1,2,-1,-2)$; for any unstable slice $H \subset H_{j} \quad(j=1,2,-1,-2)$, the diameter decay relation

$$
d(\tilde{H}) \leq \nu d(H)
$$

holds, where $\tilde{H}=P^{-1}\left(H \cap V_{k}\right), \quad(k=1,2,-1,-2)$.

The Conley-Moser conditions are sufficient conditions for establishing the topological conjugacy between the Poincare map $P$ restricted to a Cantor set in $\Sigma_{0}$, and the shift automorphism on symbols.

Let $\mathcal{W}$ be a set which consists of elements of the doubly infinite sequence form:

$$
a=\left(\cdots a_{-2} a_{-1} a_{0}, a_{1} a_{2} \cdots\right),
$$

where $a_{k} \in\{1,2,-1,-2\} ; k \in Z$. We introduce a topology in $\mathcal{W}$ by taking as neighborhood basis of

$$
a^{*}=\left(\cdots a_{-2}^{*} a_{-1}^{*} a_{0}^{*}, a_{1}^{*} a_{2}^{*} \cdots\right),
$$

the set

$$
W_{j}=\left\{a \in \mathcal{W} \mid a_{k}=a_{k}^{*}(|k|<j)\right\}
$$

for $j=1,2, \cdots$. This makes $\mathcal{W}$ a topological space. The shift automorphism $\chi$ is defined on $\mathcal{W}$ by

$$
\begin{aligned}
\chi: & \mathcal{W} \mapsto \mathcal{W}, \\
& \forall a \in \mathcal{W}, \chi(a)=b, \text { where } b_{k}=a_{k+1} .
\end{aligned}
$$

The shift automorphism $\chi$ exhibits sensitive dependence on initial conditions, which is a hallmark of chaos.

Let

$$
a=\left(\cdots a_{-2} a_{-1} a_{0}, a_{1} a_{2} \cdots\right),
$$

be any element of $\mathcal{W}$. Define inductively for $k \geq 2$ the stable slices

$$
\begin{aligned}
& V_{a_{0} a_{-1}}=P\left(H_{a_{-1}}\right) \cap H_{a_{0}}, \\
& V_{a_{0} a_{-1} \ldots a_{-k}}=P\left(V_{a_{-1} \ldots a_{-k}}\right) \cap H_{a_{0}} .
\end{aligned}
$$

By Conley-Moser condition (ii),

$$
d\left(V_{a_{0} a_{-1} \ldots a_{-k}}\right) \leq \nu_{1} d\left(V_{a_{0} a_{-1} \ldots a_{-(k-1)}}\right) \leq \ldots \leq \nu_{1}^{k-1} d\left(V_{a_{0} a_{-1}}\right) .
$$

Then,

$$
V(a)=\bigcap_{k=1}^{\infty} V_{a_{0} a_{-1} \ldots a_{-k}}
$$

defines a 2 dimensional continuous surface in $\Sigma_{0}$; moreover,

$$
\partial V(a) \subset \partial_{u} \hat{S}_{l} .
$$

Similarly, define inductively for $k \geq 1$ the unstable slices

$$
\begin{aligned}
& H_{a_{0} a_{1}}=P^{-1}\left(H_{a_{1}} \cap V_{a_{0}}\right), \\
& H_{a_{0} a_{1} \ldots a_{k}}=P^{-1}\left(H_{a_{1} \ldots a_{k}} \cap V_{a_{0}}\right) .
\end{aligned}
$$

By Conley-Moser condition (ii),

$$
d\left(H_{a_{0} a_{1} \ldots a_{k}}\right) \leq \nu_{2} d\left(H_{a_{0} a_{1} \ldots a_{k-1}}\right) \leq \ldots \leq \nu_{2}^{k} d\left(H_{a_{0}}\right) .
$$

Then,

$$
H(a)=\bigcap_{k=0}^{\infty} H_{a_{0} a_{1} \ldots a_{k}}
$$


defines a codimension 2 continuous surface in $\Sigma_{0}$; moreover,

$$
\partial H(a) \subset \partial_{s} \hat{S}_{l} .
$$

By $(5.3 ; 5.4)$ and dimension count,

$$
V(a) \cap H(a) \neq \emptyset
$$

consists of points. Let

$$
p \in V(a) \cap H(a)
$$

be any point in the intersection set. Now we define the mapping

$$
\begin{aligned}
\phi: & \mathcal{W} \mapsto \hat{S}_{l}, \\
& \phi(a)=p .
\end{aligned}
$$

By the above construction,

$$
P(p)=\phi(\chi(a))
$$

That is,

$$
P \circ \phi=\phi \circ \chi
$$

Let

$$
\Lambda \equiv \phi(\mathcal{W})
$$

then $\Lambda$ is a compact Cantor subset of $\hat{S}_{l}$, and invariant under the Poincare map $P$. Moreover, with the topology inherited from $\hat{S}_{l}$ for $\Lambda, \phi$ is a homeomorphism from $\mathcal{W}$ to $\Lambda$. Thus we have the theorem.

TheOREM 5.4 (Horseshoe Theorem). Under the generic assumptions (A1)(A3) for the perturbed nonlinear Schrödinger system (1.1), there exists a compact Cantor subset $\Lambda$ of $\hat{S}_{l}, \Lambda$ consists of points, and is invariant under P. Prestricted to $\Lambda$, is topologically conjugate to the shift automorphism $\chi$ on four symbols $1,2,-1,-2$. That is, there exists a homeomorphism

$$
\phi: \mathcal{W} \mapsto \Lambda,
$$

such that the following diagram commutes:

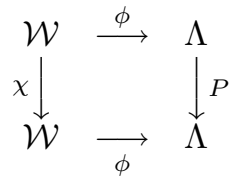

\section{Numerical Evidence for the Generic Assumptions}

6.1. Generic Assumption (A1). Figure 3 shows a numerical result of Mark Winograd. It indicates that the homoclinic orbits are indeed tangent to the $(x, y)$ plane as $t \rightarrow+\infty$. Specifically, Figure 3 (a) shows one of the homoclinic orbits, and Figure 3 (b) shows the blow-up of the neighborhood of the saddle $Q_{\epsilon}$.

6.2. Generic Assumptions (A2) and (A3). Numerical simulation of the generic assumptions (A2) and (A3) is planned for a future work. 


\section{References}

[1] Y. Li, Smale horseshoes and symbolic dynamics in perturbed nonlinear Schrödinger equations, Journal of Nonlinear Sciences 9, no.4 (1999), 363.

[2] Y. Li, Homoclinic orbits for singularly perturbed NLS, Dynamics of PDE 1, no.1 (2004), 1.

[3] N. V. Nikolenko, The method of Poincaré normal forms in problems of integrability of equations of evolution type, Russian Math. Surveys 41, no.5 (1986), 63.

[4] T. Kato, Quasi-linear equations of evolution, with applications to partial differential equations, Lecture Notes in Math., Springer 448 (1975), 25.

Department of Mathematics, University of Missouri, Columbia, MO 65211

E-mail address: cli@math.missouri.edu 